\title{
EVALUAR EN LA RED
}

\author{
Joaquín García Carrasco \\ carrascolusal.es \\ María Cruz Sánchez Gómez \\ mcsago@usal.es \\ Universidad de Salamanca
}

\author{
María Ángeles Pérez Juárez \\ mperezatel.uva.es \\ Blanca Rodríguez Pajares \\ blarodetel.uva.es \\ Universidad de Valladolid
}

\section{1. Introducción}

La evaluación es un juicio de valor sobre una realidad; en este caso la realidad a la que nos referimos es una "acción educativa" de la cual debemos obtener una serie de datos a través de determinados instrumentos y en unos tiempos establecidos. La evaluación es la que permite valorar el proceso enseñanza-aprendizaje, $\mathrm{y}$, por tanto, enseñar, aprender $\mathrm{y}$ evaluar son partes interrelacionadas.

Sin embargo, evaluar de forma objetiva, fiable y transparente no resulta fácil; existe una problemática en torno a la evaluación de aprendizajes tanto desde el punto de vista teórico como práctico, y se refiere a aspectos varios y diversos: exámenes; fines de la evaluación; programación, calificación y evaluación; desfase entre la teoría y la práctica; dificultades y límites de la evaluación; su función de control y selección.

El proceso de evaluación en el contexto lecto-escritor tiene muchas limitaciones, pero las nuevas tecnologías proporcionan posibilidades de construir modelos de evaluación que se aproximan a los criterios de evaluación. La facilidad de uso del web ha provocado su gran difusión y la creación de numerosos entornos virtuales de aprendizaje sobre esta plataforma. Pero la gran mayoría de estos entornos se limita a la presentación de contenidos, y en caso de incluir evaluación, ésta se reduce a la realización de tests. Es decir, incluyen muy pocas características del proceso de enseñanzaaprendizaje y, además, carecen de una base pedagógica.

Un problema añadido es la incompatibilidad entre los distintos entornos. La causa principal es la carencia de estándares educativos, lo que limita la interoperabilidad entre sistemas y la reutilización de recursos. Actualmente existen varias organizaciones desarrollando estándares y especificaciones de tecnología educativa, principalmente en dos aspectos: la arquitectura de los sistemas educativos y los sistemas de metainformación.

\section{La evaluación}

No existe una definición única de evaluación, pues ésta puede tener diferentes enfoques. En Rodríguez (2000) se define evaluación educativa como la medida o comprobación del grado de consecución de objetivos, lo que comporta una recogida de información para emitir un juicio de valor codificado en una calificación, con vistas a una toma de decisiones. A partir de la definición de evaluación se ve la relación directa que existe entre objetivo y evaluación, entendiendo por objetivo didáctico el resultado que esperamos que consiga el alumno como consecuencia del proceso de enseñanza-aprendizaje.

En la evaluación del aprendizaje de los alumnos encontramos los mismos elementos que en cualquier otra evaluación educativa: será necesaria una recogida de información con el fin de compararla con el criterio u objetivo de referencia, que nos permitirá formular un juicio de valor y nos servirá, a la vez, para la toma de decisiones correctoras, en su caso. Podríamos señalar que "si el aspecto a evaluar es el aprendizaje de los alumnos, el criterio será una buena formulación de objetivos en términos que permitan comparar con ellos el rendimiento del alumno" (Teijido de Suñer, 1988, 3).

Por ello, no se puede hablar de evaluación de aprendizaje sin tener en cuenta los objetivos, en este 
campo, puesto que la propia evaluación permitirá la comparación de lo conseguido con el criterioobjetivo a tener en cuenta y la toma de decisión orientada a la optimización de éste. En palabras de De la Orden $(1972,13)$ "si queremos explicar el carácter evidentemente polémico de la evaluación educativa hemos de acudir (...) al condicionamiento del proceso educativo por parte de la evaluación como determinante de los objetivos reales del aprendizaje".

\section{Tecnologías para el desarrollo de entornos virtuales de aprendizaje}

\subsection{El World Wide Web}

El World Wide Web se ha convertido en un factor cada vez más importante en la planificación de entornos informáticos para las empresas y organizaciones de todo tipo, incluidas las educativas. La razón son las ventajas que ofrece (Manola, 1998):<br>

- El software de los navegadores web está ampliamente disponible y es más barato que otras alternativas.

- La representación de la información soporta el entrelazado de toda clase de contenidos (información multimedia).

- Es de fácil acceso para los usuarios.

- La creación de contendidos es relativamente sencilla.

Sin embargo, a medida que estas organizaciones tratan de emplear el web en aplicaciones más sofisticadas, se encuentran con una serie de limitaciones. Ello es debido a que el web no fue diseñado para soportar dichas aplicaciones, sino para visualizar documentos hipermediales. Por ello se están desarrollando numerosas tecnologías para aumentar sus posibilidades. Las tendencias actuales en el Web son: incrementar su estructura y añadirle una semántica.

\section{Incrementar la estructura del WWW}

La estructura básica de datos del Web consiste hoy en día en documentos HTML hiperenlazados. Sin embargo, es algo globalmente reconocido, HTML proporciona una estructura demasiado simple como para poder soportar complejas aplicaciones. El problema reside en el hecho de que las etiquetas HTML se refieren principalmente a aspectos de presentación, sin identificar la información, de forma que ésta no puede ser procesada. En Bosak (1997) se identifican las siguientes limitaciones de HTML:

- Extensibilidad: HTML no permite a los usuarios definir sus propias marcas o atributos para ayudar a identificar el significado semántico de la información.

- Estructura: HTML no soporta la especificación de estructuras complejas, necesitadas por ejemplo, para esquemas de bases de datos o jerarquías orientadas a objetos.

- Validación: HTML no permite a las aplicaciones cliente validar la estructura de la información.

Se han desarrollado extensiones propietarias de HTML con el propósito de salvar alguno de estos problemas, pero ninguna trata con todos ellos, creando, además problemas de interoperabilidad. La dirección más importante es la que trata de integrar la tecnología orientada a objetos con la infraestructura del web. En este sentido, el web puede ser considerado como una simple forma de sistema de objetos distribuidos, en el que las páginas HTML son objetos que vienen identificados por el URL y tienen una serie de métodos invocados por servidores http, de forma que se le puede añadir un comportamiento al contenido estático de HTML.

\section{Añadir semántica al WWW}

HTML resultaba adecuado cuando las aplicaciones se limitaban a presentar páginas a los usuarios. Sin embargo, aplicaciones más complejas necesitan reconocer y procesar ciertas partes de las páginas con un específico significado semántico. En algunos casos, las aplicaciones deben reconocer información que está en un determinado formato, bien definido (por ejemplo una factura o formulario). En otros necesitan identificar cierto contenido (por ejemplo, un autor, que podría ser el de la página, o bien, el que aparece en una referencia, etc.). 
HTML se limita prácticamente a marcar cómo se presenta la información, pero lo que se necesita es un marcado semántico, en el que las marcas identifican elementos basándose en su semántica. En este sentido, hay una tendencia clara a la adopción de XML como nuevo lenguaje del Web.

\section{Recursos de aprendizaje en el WWW}

Centrándonos en el campo de educativo, los recursos de aprendizaje son los que constituyen el bloque básico, y son dos las características fundamentales que deben cumplir (CEN/ISSS 2000):

- La reusabilidad, que es importante en términos económicos y de tiempo de producción y para permitir que los recursos puedan ser desarrollados colaborativamente en una forma modular.

- La interoperabilidad, que resulta clave para los usuarios que no desean estar ligados a una tecnología de distribución específica o que desean intercambiar información.

Tanto para mejorar la reusabilidad como la interoperabilidad se hace necesario el desarrollo de estándares que permitan estructurar, describir, replicar y archivar contenido. Se definen una serie de niveles de granularidad, que varían según terminologías, y que según CEN/ISSS (2000) son los siguientes:

- Nivel 0: atomos. Separan ítems de datos, por ejemplo piezas de texto, un video clip o una imagen.

- Nivel 1: unidades de contenido. Son recursos de aprendizaje autocontenidos que no son sensibles a ser divididos.

- Nivel 2: unidades compuestas. Son grupos de unidades de contenido, que incluyen navegación dentro de un sistema de tecnología de aprendizaje.

- Nivel 3: cursos. El nivel más alto de granularidad, representa experiencias de aprendizaje con un amplio horario de acceso y ofrece regularmente acreditación.

Los módulos o unidades se describen comúnmente como "objetos de aprendizaje". No hay una definición lo suficientemente clara y, por ello, requieren ser definidos dentro de un estándar. Además, la modularización de los cursos de formación facilitará al docente llevar un registro del progreso del estudiante y hacer un reconocimiento de sus habilidades mediante procedimientos de evaluación continua.

\section{2. XML (eXtensible Markup Language)}

\section{Origen y Evolución de XML}

El origen de XML son los lenguajes de marcado. Los lenguajes de marcado describen la estructura de los distintos tipos de documentos, basándose en la utilización de etiquetas, marcas, o tags que se sitúan al comienzo y final del documento y de cada estructura del documento definida, identificando su función y naturaleza. Así, por ejemplo, podríamos definir dentro de un documento secciones, títulos, tablas, párrafos, etc. La finalidad es, una vez marcado el documento, que éste pueda ser tratado desde cualquier sistema (Lander, 1999).

Fue en los años 60 cuando IBM desarrolló GML (Generalized Markup Language, lenguaje de marcas generalizado) para resolver sus problemas de publicación, incluyendo el tratamiento de documentos de todos tipos, desde manuales y anuncios de prensa hasta contratos legales y especificaciones de proyectos (Connolly, 1997).

El GML inicialmente diseñado por Charles F. Goldfard en IBM en 1969 se continuó desarrollando hasta convertirse en la norma internacional ISO 8879 en 1986, conocida como SGML (Standard Generalized Markup Language, lenguaje de marcado generalizado estándar) [ISO 8879:1986]. Desarrollado para definir y usar formatos de documento portables, SGML se diseñó para ser lo suficientemente formal como para permitir pruebas de validez de documentos, lo suficientemente estructurado como para tratar documentos complejos y lo suficientemente extensible como para soportar la gestión de grandes cantidades de información (Connolly, 1997). SGML es un metalenguaje que permite definir lenguajes de marcas, tipo HTML o RTF (Rich Text Format, formato de texto enriquecido). La definición de la estructura y contenido del documento se realiza 
en una DTD (Document Type Definition, definición del tipo de documento), donde se especifican los elementos que conformarán ese tipo de documento y cómo tienen que estar organizados (Bravo, 1999).

En 1989, el investigador del CERN (Centro Europeo de Investigación Nuclear) Tim Berners-Lee propuso compartir información utilizando documentos de texto de hiperenlace y a partir de un simple tipo de documento según la norma SGML desarrolló una versión de hipertexto denominada HTML (Hiper Text Markup Language, lenguaje de marcado de hipertexto).

A medida que la popularidad del web fue aumentando, HTML se fue desarrollando según los intereses de los fabricantes de navegadores. Éstos fueron añadiendo etiquetas propietarias, dirigidas principalmente a controlar la presentación de los documentos para que éstos estuviesen perfectamente formateados, sin permitir diferencias importantes entre distintos visualizadores. El resultado fue la generación de extensiones de HTML incompatibles y que se alejaban del ideal inicial de marcar la información de acuerdo únicamente con el significado. El W3C (World Wide Web Consortium, Consorcio de la Web) constituido en 1994 y presidido por el mismo Tim Berners-Lee, decidió tomar medidas con el fin último de asegurar la interoperabilidad en el Web. Desarrolló de un lenguaje de hoja de estilos específico de HTML llamado CSS (Cascading Style Sheets, hojas de estilo en cascada), capaz de describir el estilo de los documentos HTML, sin interferir con su marcado específico.

Asimismo, comenzó el desarrollo de XML (eXtended Markup Language) como un metalenguaje (no un lenguaje como HTML) subconjunto de SGML, más fácil de escribir, interpretar e implementar que SGML. Fue diseñado para que se pudieran crear más rápida y eficientemente lenguajes de marcas propios que se ajustaran a las necesidades de cada cual. XML es, por tanto, un metalenguaje, y como tal, no posee sus propias etiquetas, sino que es el propio autor el que las crea dependiendo del contenido del documento. De esta manera, XML supera las limitaciones de HTML, que mezcla estructura y presentación, y que no es extensible.

Recientemente el W3C ha definido xHTML (eXtensible Hyper-Text Markup Language, lenguaje de marcado de hipertexto extensible), que es la reformulación de la última versión estandarizada de HTML, pero dentro de las normas de XML; volviendo de esta manera a las ideas originales de separar estructura y contenido de la presentación de un documento.

\section{Estructura de XML}

La estructura lógica proporcionada por XML es una estructura en árbol, fácil de desarrollar e implementar. Con XML se tiene una visión jerárquica de los documentos, donde los elementos que constituyen el árbol son definidos por el propio diseñador de XML dependiendo del contenido del documento. XML proporciona el estándar para codificar el contenido, el significado y el esquema de dicho documento, que puede ser desde un simple texto, hasta un registro de una base de datos o una presentación gráfica. Lo importante es que XML se centra en una declaración más precisa de los contenidos, sin entrar en su presentación. Todo esto facilita la integración de datos procedentes de distintas fuentes y unos resultados en las búsquedas más significativos, con una información estructurada uniforme e independiente de las aplicaciones (Microsoft Corporation, 2000).

Para definir la jerarquía un documento XML, se utiliza el DTD (Document Type Definition), que especificando los elementos que puede haber en un documento, así como su relación entre ellos, los posibles valores, etc. (Barbero, 1999). Se puede decir que un DTD es la definición exacta de la gramática de un documento, con el objetivo de que se genere un código correcto sin errores (Montero, 2000).

Un documento debe seguir las normas de la especificación XML para estar bien formado (es decir, para ser un documento XML). Si además el documento está sujeto a los elementos definidos en un DTD, algo que no es necesario, entonces se dirá que el documento es válido. Por tanto, un documento XML debe estar bien formado pero no tiene por qué ser válido.

\section{Tecnologías asociadas a XML}

Con XML, ciertos aspectos quedan incompletos con los lenguajes existentes, por lo que se están 
desarrollando unos nuevos, como son XSL, para la representación de los documentos XML, y XLINK y XPOINTER para definir los hiperenlaces.

\subsection{Metainformación}

La metainformación, o metadatos es información acerca de la información. El término se refiere a cualquier información que se use para identificar, describir, o localizar recursos electrónicos dentro de la red. Puede describir, por ejemplo, el autor de un trabajo, la fecha de publicación, u otros detalles que ayudarán posteriormente a su clasificación o recuperación.

El principal objetivo de la metainformación es mejorar la gestión y recuperación de información. El web es una gran fuente de información, pero sin ningún orden. Para realizar búsquedas se utilizan los motores de búsqueda, que buscan por todo el mundo similitudes de la palabra o frase introducida con el contenido de las etiquetas de metainformación que llevan las páginas web. El resultado suele ser una mezcla de enlaces relevantes con otros irrelevantes (éstos últimos muchas veces en gran cantidad). Y es que Internet, y más particularmente el web, no fue diseñado como soporte de publicaciones ordenadas o de búsqueda de información como lo son las bibliotecas; en ella no sólo se encuentran libros y revistas, sino también vídeos, imágenes noticias, conversaciones transcritas, etc. Es, por tanto, necesaria la descripción de recursos para que la web evolucione de su estado actual a otro donde los recursos estén organizados, catalogados y se puedan buscar y encontrar de manera eficiente. El objetivo final es construir una "web semántica", que incluya documentos que describan de forma explícita las relaciones entre distintos recursos y que contengan información semántica que permita su procesado automático (Berners-Lee, 1999).

La arquitectura de la metainformación se representa como un conjunto declaraciones <nombre de atributo, valor del atributo $>$. Para representar todos estos campos e indicar sus valores se puede utilizar HTML, mediante el uso de las etiquetas $<$ META $>$ y $<$ SPAN $>$. Sin embargo, no era éste el objetivo original de HTML y su uso se limita a declarar propiedades globales que se refieren al documento entero. Mejor opción es XML, que permite estructurar las páginas web como árboles etiquetados, donde las etiquetas pueden ser escogidas para reflejar la semántica específica del área de la información.

La descripción del recurso deberá ser consistente, de forma que las declaraciones puedan ser tratadas de forma genérica tanto por personas como por máquinas (Berners-Lee, 1997). También deberá ser fácil de crear para que cualquier autor pueda fácilmente describir los contenidos de sus recursos, y que éstos sean más útiles y accesibles.

\section{Estandarización y especificación en tecnología educativa}

\section{1. Necesidad de estandarización}

Uno de los principales problemas a la hora de desarrollar materiales de aprendizaje multimedia de alta calidad es el alto coste, por lo que la posibilidad de reutilización se convierte en un requisito clave. Pero para ello es necesario elaborar una serie de estándares que garanticen esa posibilidad de reutilización.

Por otro lado, la adopción de este tipo de estándares que garanticen la interoperabilidad puede significar un nuevo paradigma para el mercado de productos de formación, pues al permitir la integración de materiales con distintos niveles de granularidad, se facilita la creación de programas de teleformación a partir de repositorios comunes de información, que pueden contener materiales producidos por distintos proveedores.

Estos componentes u Objetos de Aprendizaje (Learning Objects), que no son más que contenido que puede distribuirse a través del web, pueden ser reutilizados y combinados de manera flexible para que se adapte a las necesidades individuales, lo que aumenta la eficiencia en el desarrollo y distribución de cursos, además de reducir costes.

La definición de estándares abiertos permitirá conseguir un entorno en el que se cumplan todos estos requisitos. Y aunque algunos puntos puedan ser satisfechos por estándares más genéricos de Tecnologías de la Información y la Comunicación, otros muchos deberán ser desarrollados para 
satisfacer requisitos específicos de tecnología educativa, en todos los aspectos que ésta conlleva.

\section{2. Iniciativas de estandarización y especificación}

Existen numerosas organizaciones que están desarrollando estándares y especificaciones de tecnología educativa. Hay que señalar aquí que estándar y especificación no es lo mismo, sino que los estándares sólo pueden proceder de instituciones acreditadas como tal y deben, además, seguir un proceso especial. Son los casos de IEEE, CEN/ISSS e ISO.

Aunque nos centraremos en los trabajos de dos organizaciones, IEEE LTSC e IMS, en la siguiente tabla se citan otras que igualmente están trabajando en tecnología educativa:

\begin{tabular}{|c|c|}
\hline Organización - Proyecto & Breve Descripción \\
\hline $\begin{array}{l}\text { AICC (Aviation Industry CBT } \\
\text { Committee) }\end{array}$ & $\begin{array}{l}\text { Iniciativa de CBT (Computer-Based Training). Es un foro abierto de } \\
\text { profesionales de la educación que elabora una serie de normas para fomentar al } \\
\text { interoperabilidad de la tecnología educativa (AICC, 2000). }\end{array}$ \\
\hline ADL (Advanced Distributed Learning) & $\begin{array}{l}\text { Iniciativa del Departamento de Defensa (DoD) y la Oficina de Política } \\
\text { Científica y Tecnológica de la Casa Blanca (OSTP). Su objetivo es asegurar el } \\
\text { acceso a una educación y unos materiales de formación de alta calidad que se } \\
\text { puedan adaptar a las necesidades individuales del estudiante (ADL, 2000). }\end{array}$ \\
\hline $\begin{array}{l}\text { GEM (Gateway to Educational } \\
\text { Materials) }\end{array}$ & $\begin{array}{l}\text { Proyecto de la Biblioteca Nacional de Educación de los Estados Unidos como } \\
\text { respuesta a un mandato del presidente para el desarrollo de proyectos que } \\
\text { potencien la Sociedad de la Información en todos los colegios (GEM, 2000). }\end{array}$ \\
\hline \begin{tabular}{|l|} 
ARIADNE (Alliance of Remote \\
Instructional Authoring and Distribution \\
Networks for Europe)
\end{tabular} & $\begin{array}{l}\text { Proyecto de investigación y desarrollo tecnológico, perteneciente al marco } \\
\text { "Telemática para la Educación y la Formación" de la Unión Europea } \\
(\text { ARIADNE, 2000). }\end{array}$ \\
\hline $\begin{array}{l}\text { PROMETEUS (PROmoting } \\
\text { Multimedia access to Education and } \\
\text { Training in EUropean Society) }\end{array}$ & $\begin{array}{l}\text { Iniciativa de la Unión Europea, cuyo objetivo es constituir un foro abierto en el } \\
\text { que se debatan cuestiones relacionadas con temas como la construcción de } \\
\text { entornos de aprendizaje basados en estándares abiertos o repositorios de } \\
\text { conocimiento de acceso público (PROMETEUS, 2000). }\end{array}$ \\
\hline $\begin{array}{l}\text { GESTALT (Getting Educational } \\
\text { Systems Talking Across Leading-Edge } \\
\text { Technologies) }\end{array}$ & $\begin{array}{l}\text { Proyecto de ACTS (Advanced Communications Technologies and Services). } \\
\text { Su objetivo principal es el de producir especificaciones públicas de interfaces } \\
\text { abiertos y estructuras de metainformación (GESTALT, 2000). }\end{array}$ \\
\hline
\end{tabular}

Tabla 1 Iniciativas de estandarización y especificación

\section{IEEE LTSC (Learning Technology Systems Committee).}

El LTSC es uno de los Comités de Estandarización del IEEE cuya misión es el desarrollo de estándares técnicos, prácticas recomendadas y guías que faciliten el desarrollo, despliegue, mantenimiento e interoperación de implementaciones de componentes y sistemas de tecnología educativa y de formación (LTSC, 2000).

Dentro del IEEE LTSC, el grupo de trabajo de Actividades Generales tiene la función, entre otras, de desarrollar un Modelo de Referencia de Arquitectura. Actualmente está definido un estándar en fase de revisión conocido como Especificación LTSA (Learning Technology Systems Architecture) (IEEE, 2000). No se trata de una propuesta de diseño de un sistema concreto sino que establece un marco general bien definido para el análisis, diseño, implementación y evaluación de este tipo de Entornos (IEEE, 2001).

Otro de los grupos de trabajo del IEEE LTSC es el de información y metainformación, que ha desarrollado el estándar LOM (Learning Objects Metadata). Este estándar especifica la sintaxis y la semántica de la metainformación de recursos educativos, definidas como: "aquellos atributos necesarios para la descripción completa y adecuada de un objeto educacional" (IEEE, 2001).Se entiende por objeto educacional una entidad, digital o no digital, que puede ser usada, reutilizada o referenciada durante cualquier actividad de aprendizaje basada en la tecnología.

Se trata de una iniciativa promovida por EDUCAUSE, asociación americana dedicada a la 
promoción de las tecnologías educativas; es un consorcio internacional formado por organizaciones educativas, comerciales y gubernamentales (IMS, 2000). Su objetivo principal es el desarrollo de especificaciones para facilitar la interoperabilidad entre aplicaciones de aprendizaje distribuido. Actualmente tiene definidas especificaciones en las áreas de metainformación, empresa, empaquetado de contenidos y evaluación.

Su especificación de metainformación está basada completamente en el estándar IEEE LOM. Es la única organización que tiene definida además una especificación que trata los tests y las pruebas objetivas; se denomina Question \& Test Interoperation (QTI) y describe la estructura de datos de preguntas (ítems) y tests (assessments) (Smythe, 2001).

\section{Módulos de evaluación en los entornos virtuales de aprendizaje actualmente desarrollados}

En el momento actual existen numerosos espacios virtuales de promoción del conocimiento, aunque no todos ellos incluyen módulos de evaluación, como es el caso de Learning Space, uno de los entornos más extendidos.

Examinando numerosos entornos de evaluación, todos ellos están basados en la realización de tests por parte del alumno. Incluyen herramientas para que el profesor diseñe y cree de tests, y para que el alumno los resuelva y consulte sus resultados.

En la tabla se recogen características de distintas herramientas de evaluación. La mayoría de ellas están basadas en el web. Suelen incluir realimentación automática, de forma que el alumno puede consultar resultados una vez realizado el test, sin incluir las respuestas abiertas. También, en su mayoría, ofrecen la posibilidad de crear de informes con estadísticas sobre los resultados obtenidos por el alumno.

\begin{tabular}{|c|c|c|}
\hline Herramienta - URL & Tipos de Preguntas & Otras Características \\
\hline $\begin{array}{c}\text { Course Test Manager } \\
\text { www.course.com }\end{array}$ & \begin{tabular}{|l} 
Elección Múltiple \\
Verdadero / Falso \\
Respuesta Limitada \\
Pregunta Abierta
\end{tabular} & $\begin{array}{l}\text { Planificación } \\
\text { Control del Tiempo } \\
\text { Realimentación Automática } \\
\text { Creación de Informe }\end{array}$ \\
\hline $\begin{array}{c}\text { eduTest.com's Online Assessment } \\
\text { System } \\
\text { www.edutest.com }\end{array}$ & \begin{tabular}{|l} 
Elección Múltiple \\
Verdadero / Falso
\end{tabular} & \begin{tabular}{|l} 
Planificación \\
Realimentación Automática \\
Creación de Informe
\end{tabular} \\
\hline $\begin{array}{l}\text { ESATEST 2000 } \\
\text { www.esatest.com }\end{array}$ & \begin{tabular}{|l} 
Elección Múltiple \\
Verdadero / Falso \\
Respuesta Limitada \\
Pregunta Abierta
\end{tabular} & $\begin{array}{l}\text { Planificación } \\
\text { Control de Tiempo } \\
\text { Pistas } \\
\text { Realimentación Automática } \\
\text { Permite exportar a Excel } \\
\end{array}$ \\
\hline $\begin{array}{l}\text { HITRETURN } \\
\text { www.hitreturn.com }\end{array}$ & \begin{tabular}{|l} 
Elección Múltiple \\
Verdadero / Falso \\
Respuesta Limitada \\
Marcar Imagen \\
\end{tabular} & $\begin{array}{l}\text { Creación de Informe } \\
\text { No basado en Web. }\end{array}$ \\
\hline $\begin{array}{c}\text { inQsit } \\
\text { www.bsu.edu/inqsit// }\end{array}$ & \begin{tabular}{|l} 
Elección Múltiple \\
Verdadero/Falso \\
Respuesta Múltiple \\
Emparejar \\
Selección \\
Respuesta Limitada \\
Marcar Imagen \\
\end{tabular} & $\begin{array}{l}\text { Planificación } \\
\text { Control de Tiempo } \\
\text { Pistas } \\
\text { Realimentación Automática }\end{array}$ \\
\hline $\begin{array}{c}\text { LXR Test } \\
\text { www.lxrtest.com }\end{array}$ & $\begin{array}{l}\text { Elección Múltiple } \\
\text { Verdadero/Falso } \\
\text { Emparejar } \\
\text { Respuesta Numérica } \\
\text { Selección } \\
\text { Pregunta Abierta } \\
\end{array}$ & Planificación \\
\hline $\begin{array}{c}\text { MicroTest Pro - eQuiz } \\
\text { equiz.classmanager.com/startquiz.asp }\end{array}$ & \begin{tabular}{|l} 
Elección Múltiple \\
Verdadero/Falso
\end{tabular} & Planificación \\
\hline
\end{tabular}




\begin{tabular}{||l||l||l||}
\hline \multicolumn{1}{|l||}{\begin{tabular}{l|l||} 
MicroTest Pro - WebTest \\
www.chariot.com
\end{tabular}} & $\begin{array}{l}\text { Pregunta Abierta } \\
\text { Elección Múltiple } \\
\text { Verdadero/Falso } \\
\text { Pregunta Abierta }\end{array}$ & $\begin{array}{l}\text { Planificación } \\
\text { Control de Tiempo } \\
\text { Permite incluir gráficos, ecuaciones, } \\
\text { símbolos Realimentación Automática } \\
\text { Creación de Informe }\end{array}$ \\
\begin{tabular}{rl|l} 
PC University \\
www.teachingtech.com/pcu.com
\end{tabular} & $\begin{array}{l}\text { Elección Múltiple Respuesta Limitada } \\
\text { Pregunta Abierta Emparejar }\end{array}$ & $\begin{array}{l}\text { Pistas } \\
\text { Información Multimedia } \\
\text { Realimentación Automática } \\
\text { Creación de Informe }\end{array}$ \\
\hline \hline $\begin{array}{c}\text { The Quiz Engine } \\
\text { Bytesinteractive.com/index.html }\end{array}$ & $\begin{array}{l}\text { Elección Múltiple } \\
\text { Verdadero / Falso }\end{array}$ \\
\hline \hline
\end{tabular}

Tabla 2 Herramientas de Evaluación y sus características

\section{Conclusiones}

Se pueden señalar las siguientes conclusiones:

- La evaluación es una parte imprescindible dentro del proceso enseñanza- aprendizaje, como medida de la consecución de los objetivos de aprendizaje por parte del alumno, y, también, como control de la calidad de dicho proceso. Sin embargo, los instrumentos de evaluación actuales presentan muchas limitaciones en el contexto lecto-escritor, que pueden ser superadas, en parte, por las nuevas tecnologías de la información y la comunicación.

- Están surgiendo nuevas tecnologías que permiten la construcción de modelos más completos que se aproximan más a los criterios de evaluación. En su mayoría son tecnologías orientadas a objetos, que proporcionan mayores ventajas que las tecnologías tradicionales. De igual forma, el Web está evolucionando hacia una estructura modular, siguiendo esa misma filosofía. El Web es un espacio universal de información, pero se trata de convertirlo en un espacio universal de conocimiento.

- Una de estas nuevas tecnologías que permite convertir el Web en un espacio universal de conocimiento es XML, que aunque no es tan nuevo, sí es ahora cuando está empezando a utilizarse. XML surge ante las limitaciones de HTML para definir una estructura semántica de los contenidos. XML permite, de forma sencilla, estructurar la información, separando contenido y presentación. De esta forma, XML puede utilizarse como:

- Un formato de descripción de datos

- Un formato de intercambio de datos

- Un formato de almacenamiento de datos

- Un formato de almacenamiento de documentos

- Existe una carencia de estándares referentes a tecnología educativa, lo que provoca una diversidad de entornos no interoperables, es decir, incompatibles, y con una diversidad de contenidos. Hay una ausencia de estrategias comunes de diseño, desarrollo e implementación. El desarrollo y la adopción de estos estándares proporcionarán:

- Una guía en el diseño y la implementación de entornos tecnológicos de enseñanzaaprendizaje.

- Una flexibilización de los materiales y los entornos, pudiendo ser fácilmente adaptados a diferentes contextos y plataformas.

- Una disminución de los costos de producción.

- Una disminución de los tiempos de producción.

- Una mayor oferta de aprendizaje en entornos tecnológicos.

- Diversas organizaciones están trabajando en el desarrollo de estándares. Entre ellas cabe destacar el IEEE, concretamente su comité LTSC (Learning Technology Stadard Committee), e IMS. En todos los casos, se pretende garantizar:

- Accesibilidad: posibilidad de acceder a recursos educativos desde un sitio remoto y poder distribuirlos a otros muchos sitios.

- Interoperabilidad: posibilidad de usar recursos educativos desarrollados en un sitio con un conjunto de herramientas o plataforma en otra plataforma en otra localización con otras herramientas.

- Durabilidad: recursos educativos que no requieran ser rediseñados cuando cambia la 
tecnología base.

- Reusabilidad: diseño de recursos educativos que pueden ser incorporados en múltiples aplicaciones.

- El trabajo realizado en estandarización de tecnología educativa, es escaso, y los estándares publicados son bastante limitados.

- Se han desarrollado una serie de entornos, pero centrados casi exclusivamente en la presentación de contenidos. No consideran todo el proceso enseñanza - aprendizaje, y, en caso de incluir un módulo de evaluación, éste prácticamente se limita a los tests como único instrumento de evaluación. Además, carecen de base pedagógica, y no se adaptan al alumno.

\section{Bibliografia}

ADLnet (2000) Advanced Distributed Learning Network. http://www.adlnet.org/.

AICC (2000) Aviation Industry CBT Committee. http://www.aicc.org/.

ARIADNE (2000) European Union Commission, and, for Swiss contractors, by the Swiss Federal Office for Education and Science (OFES). Alliance of Remote Instructional Authoring and Distribution Networks for Europe. http://ariadne.unil.ch/.

BERNERS-LEE, T. (1997) Metadata Architecture. http://www.w3.org/DesignIssues/Metadata BERNERS-LEE, T.; CONNOLLY, D. y SWICK, R (1999) Web Architecture: Describing and Exchanging Data. http://www.w3.org/199/04a/WebDat.
BRAVO,
J. (1999)
eXtensible
Markup
Language.

http://html.programacion.net/xml/htmdssl/capitulo1/capitulo1.htm.

CEN/ISSS (1998) Information Society Standardization System.

http://www.cenorm.be/isss/workshop/lt/

CONNOLLY, D.; KHARE, T. y RIFKIN, A. (1997) The Evolution of Web Documents: The Ascent of XML. http://www.cs.caltech.edu/ adam/papers/xml/ascent-of-xml.html.

DE LA ORDEN, A. (1985) "Modelos de evaluación universitaria", Revista Española de Pedagogía, 169-170, 521-537.

DEROSE, S.; MALER, E.; ORCHAD, D. y TRAFFORD, B (2000) XML Linking Language (XLink) Version 1.0. http://www.w3.org/TR/2000/CR-xlink-20000703/.

GEM (2000) The Gateway to Educational Materials. http://www.geminfo.org/.

GOLDFARB F. y PRESCOD, P. (1999) Manual de XML. Madrid., Prentice.

IEEE (2001) Draft Standard for Learning Technology-Learning Technology Systems Architecture (LTSA) [IEEE P1484.1/D7].

IEEE (2000) Draft Standard for Learning Object Metadata. [IEEE P1484.12 D6.1]

IMS (2000) IMS Global Learning Consortium, Inc. http://www.imsproject.org.
LANDER,
R. The
Search
for
Metadata.

http:/pdbeam.uwaterloo.ca/ rlander/Metadata/metadata.html.

MANOLA, F. (1998) Towards a Web Object Model. http://www.objs.com/OSA/wom.htm.

MICROSOFT CORPORATION (2000) MSDN online Library. http://edutool.com/ltsa.

MONTERO, R. (2000) XML, el lenguaje universal. http://www.ramon.org/xml/intro_xmlxml_css.xml.

PROMETEUS (2000) Promoting Multimedia access to Education and Training in European Society, http://www.prometeus.org/.

RODRÍGUEZ, T.; ÁLVAREZ, L.; CADRECHA, M.; HERNÁNDEZ, J.; LUENGO, M.; MUÑIZ, J.; ORDÓÑEZ, J. y SOLER, E. (2000) La Evaluación en el Aula. Barcelona, Ediciones Nobel.

SMITHE (2001) IMS Question \& Test Interoperability: ASI Information Model Specification. http://www.imsproject.org/question/qtinfo03.html.

TEIJIDO DE SUNER, E. (1988) Evaluación del aprendizaje. Buenos Aires, Stella.

(C) Ediciones Universidad de Salamanca 